\title{
HIGH-PERFORMANCE FLEXIBLE FORCE AND TEMPERATURE SENSING ARRAY WITH A ROBUST STRUCTURE
}

\author{
MIN-SEOK KIM* \\ Center for Mass-Related Quantities, Korea Research Institute of Standards and Science, Science Town \\ Daejeon, 305-340, Republic of Korea \\ minsk@kriss.re.kr \\ HAN-WOOK SONG \\ Center for Mass-Related Quantities, Korea Research Institute of Standards and Science, Science Town \\ Daejeon, 305-340, Republic of Korea \\ hanugi16@kriss.re.kr \\ YON-KYU PARK \\ Center for Mass-Related Quantities, Korea Research Institute of Standards and Science, Science Town \\ Daejeon, 305-340, Republic of Korea \\ ykpark@kriss.re.kr
}

\begin{abstract}
We have developed a flexible tactile sensor array capable of sensing physical quantities, e.g. force and temperature with high-performances and high spatial resolution. The fabricated tactile sensor consists of $8 \times 8$ force measuring array with $1 \mathrm{~mm}$ spacing and a thin metal (copper) temperature sensor. The flexible force sensing array consists of sub-millimetre-size bar-shaped semi-conductor strain gage array attached to a thin and flexible printed circuit board covered by stretchable elastomeric material on both sides. This design incorporates benefits of both materials; the semiconductor's high performance and the polymer's mechanical flexibility and robustness, while overcoming their drawbacks of those two materials. Special fabrication processes, so called "drytransfer technique" have been used to fabricate the tactile sensor along with standard microfabrication processes.

Keywords: Flexible tactile sensor; force sensing array; polymer and silicon micro-machining; thin temperature sensor.
\end{abstract}

\section{Introduction}

The sense of touch provides us with important information about our surroundings. We can manipulate and recognize objects and even be aware of harmful situations with the help of sensory data for touch sensing provided by our skin, which is located at the

This is an Open Access article published by World Scientific Publishing Company. It is distributed under the terms of the Creative Commons Attribution 3.0 (CC-BY) License. Further distribution of this work is permitted, provided the original work is properly cited.

${ }^{*}$ Corresponding author. 
interface between our bodies and the outside world. Similarly, tactile sensing has the potential to be used in robotic systems for their advanced and complex tasks ${ }^{1-3}$.

For the last few decades, a lot of tactile sensing technologies have been introduced for robotic or medical applications. However, their further advances toward tactile sensors on a par with human skin have been hampered by lack of available technologies to fill all of the requirements at the same time that human skin has, such as flexibility, mechanical and chemical robustness, high-sensitivity, high-repeatability, multi-modal measurements (pressure, vibration, temperature, slip detection), deployment of many sensing elements, covering any shaped surface with 3-dimensional curvature (e.g. a sphere), and sensor addressing for arrays.

In our previous article $e^{4,5}$, we introduced a new concept of the flexible tactile sensor array that has the potential to fill most of needs described above and demonstrated the feasibility of using this concept by fabricating a prototype $5 \times 5$ force-sensing array with commercial semi-conductor stain gauges. Our new approach is using two materials; inorganic silicon and polymers that have complementary material properties to each other. In this paper, we report an improved tactile sensing array that has $8 \times 8$ force-sensing elements spaced $1 \mathrm{~mm}$ apart and temperature sensing capability.

\section{Sensor Structure}

The structure of the tactile sensing device is basically same as the prototype device reported before ${ }^{4,5}$. The sensing elements (semiconductor strain gauges) are equally distributed with a spacing of $1 \mathrm{~mm}$ on the $25 \mu \mathrm{m}$-thick plastic substrate with sensing metal lines. Both sides of the plastic substrate are covered with $0.5 \mathrm{~mm}$-thick elastomeric rubber layers. The rubber layer plays a role of not only protection of circuits from contamination due to contact but also spring elements being deformed by external forces. Force distribution can be obtained by reading the change of resistance of each gauge through a scan of row and column signal lines.

In order to sense temperature simultaneously, an additional layer is incorporated into the device. The temperature-sensing layer consists of $12.5 \mu \mathrm{m}$-thick plastic substrate and a temperature sensor made of $300 \mathrm{~nm}$-thick copper layer patterned on it. The solid copper has a temperature coefficient of resistance of approximately $0.39 \% / \mathrm{K}$. The temperature sensing layer is located on top of the force sensing layer in order to accept heat flow more quickly from the contact surface. Although, the temperature sensor can be influenced by external force distribution due to its flexibility, the change in resistance due to mechanical strain would be negligible compared to that due to $1 \mathrm{~K}$ temperature change, i.e., the maximum relative change in resistance due to mechanical strain can be calculated to be approximately $0.02 \%$ (10 times smaller than the change in resistance due to $1 \mathrm{~K}$ change in temperature) assuming the maximum strain and the gauge factor of copper is 0.01 and 2.0, respectively. The plan and exploded view of the tactile sensor are shown in Fig. 1(a) and 1(b), respectively. 

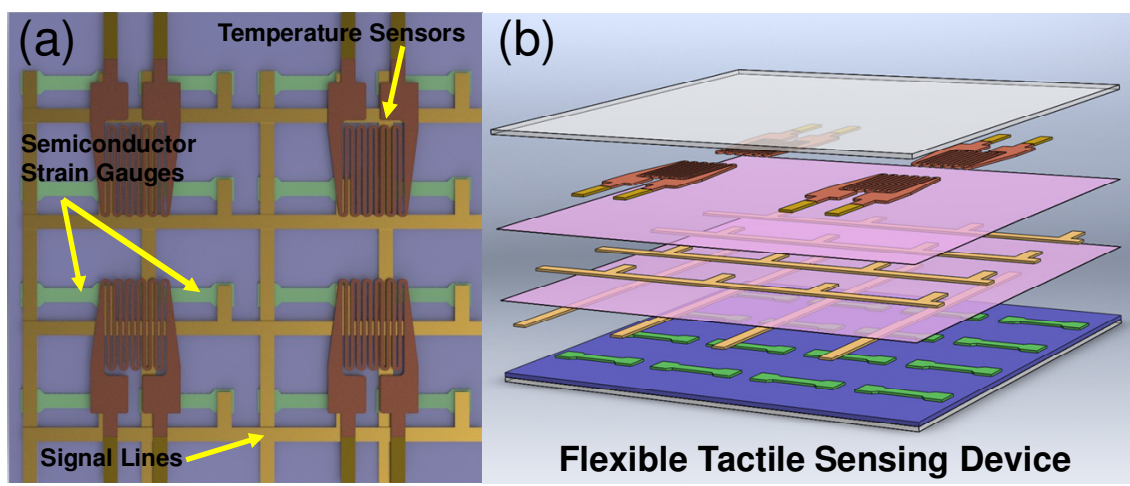

Fig. 1. The schematic of the tactile sensing device: (a) a plane view, (b) an exploded view.

\section{Fabrication}

The tactile sensing device is comprised of the force-distribution sensing layer and the temperature sensing layer. These two layers were fabricated separately for manufacturing convenience and clued together to form one device.

\subsection{Fabrication of the flexible force-distribution sensing layer}

The schematic of the fabrication process and the fabricated layer are shown in Fig. 2. The fabrication starts with silicon-on-insulator (SOI) wafers with a $100 \mathrm{~nm}$-thick top silicon layer. The top silicon layer was doped using boron with a concentration of $1 \times 10^{19}$ ions $/ \mathrm{cm}^{3}$ through an ion-implantation process so that the top silicon layer has a piezoresistive property. Then, the doped silicon layer was transferred to $25 \mu \mathrm{m}$-thick plastic substrate (polyimide film) by so-called 'dry-transfer-printing' technique ${ }^{6}$.

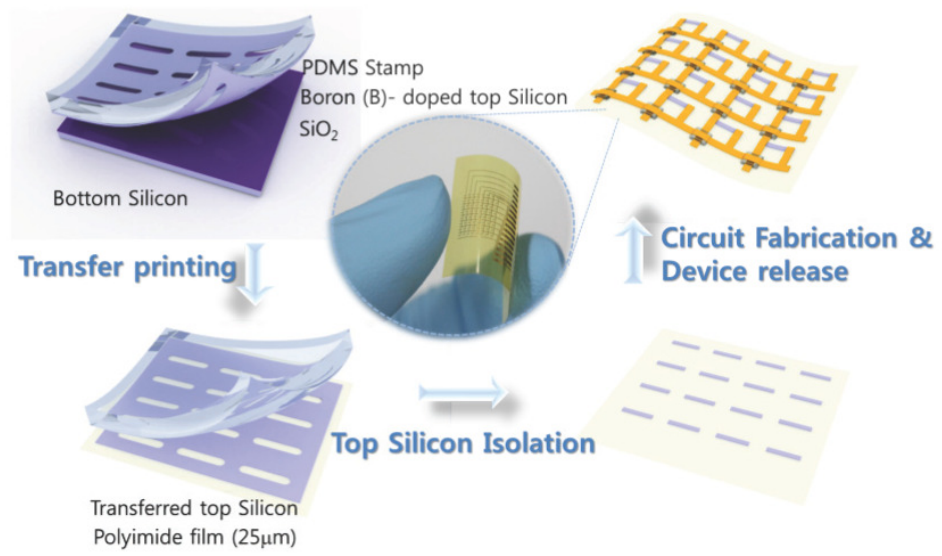

Fig. 2. The schematic of the fabrication process: the inset in the center shows a fabricated force-distribution sensing layer. 
The transferred silicon layer was etched to form bar-shaped $8 \times 8$ gauge arrays. The arrayed strain gauges are interconnected by 8 row and 8 column signal lines. The signal lines were formed by depositing metal layers ( $5 \mathrm{~nm} \mathrm{Cr}$ layer and $100 \mathrm{~nm}$ Au layer) using e-beam evaporating process and the following etching process. For passivation at cross area between row and column signal lines, polymer islands made of SU-8 with a thickness of $500 \mathrm{~nm}$ have been formed at cross area. The exploded view of the forcedistribution sensing layer is depicted in Fig. 3.

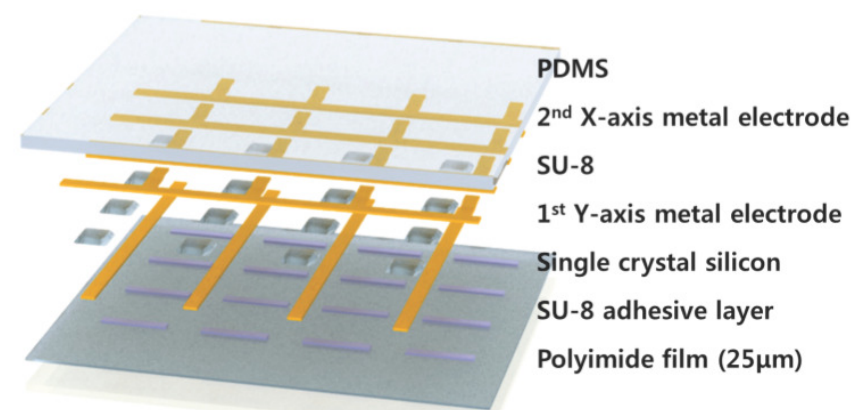

Fig. 3. The exploded view of the fabricated force-distribution sensing layer.

\subsection{Fabrication of the flexible temperature sensing layer}

The flexible temperature sensing layer was fabricated using a simple metal deposition process. We prepared a substrate by attaching $12.5 \mu \mathrm{m}$-thick polyimide film on an 8-inch silicon wafer using UV-activated glue. Then, a copper layer was formed on the substrate with a thickness of approximately $300 \mathrm{~nm}$ through a metal sputtering process. The deposited copper layer was patterned into a copper grid like conventional strain gauges so that the nominal resistance of the temperature sensor is approximately $10 \mathrm{k} \Omega$. Fig. 4(a) and 4(b) show a mask pattern for lithography and a fabricated temperature sensor, respectively.

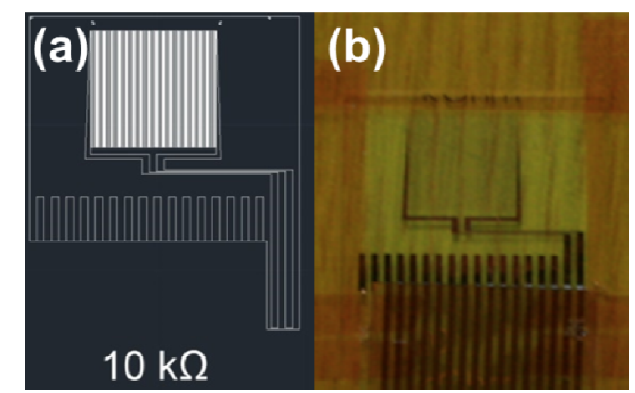

Fig. 4. The temperature sensor: (a) a mask pattern for lithography, (b) a photo of the fabricated temperature sensing layer. 
The temperature sensing layer was glued on the force-distribution sensing layer using a bond for strain gauges. Both sides of the bonded device are covered with $0.5 \mathrm{~mm}$-thick elastomeric rubber substrates (PDMS, polydimethylsiloxane) for protection. As described previously, the bottom substrate also plays a role of a spring element for force distribution sensing. Equal thickness of rubber substrates on both sides can reduce mechanical stress applied on the device when the device is bent because the neutral axis is placed close to the device layer. The final tactile sensing device is shown in Fig. 5(a) and 5(b).

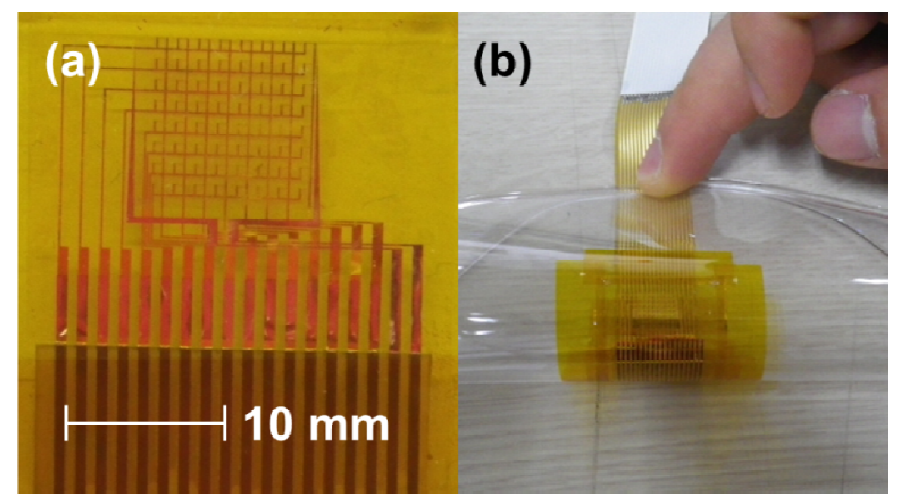

Fig. 5. The tactile sensing device: (a) a final fabricated device, (b) a photo of the fabricated temperature sensing layer.

\section{Performance Test}

The fabricated tactile sensing device was tested for two categories. One is to test measuring capabilities of force distribution and the other is to test temperature measuring performances. A video clip showing its performances will be provided during our presentation at the conference.

\subsection{Test of the force-distribution sensing layer}

The force-distribution sensing layer was tested with with an experimental setup consisting of a precision balance with $0.01 \mathrm{~g}$ resolution and an indenter with a tip diameter of $1 \mathrm{~mm}$ as shown in Fig. 6. We applied stepwise forces up to approximately $120 \mathrm{~g}$ (i.e., approximately $1 \mathrm{MPa}$ ) on each taxel (tactile pixel) by indenting the upper PDMS substrate, underneath which a strain gage was located, with the tip while measuring the contact force with the balance and simultaneously acquiring the signal output from the taxel through the signal processing $\operatorname{system}^{4,5}$ at each step. The loads were sequentially loaded up and down repeatedly five times in order to observe the repeatability and the hysteresis of the taxel output. We repeated this test for 17 taxels selected among total 64 taxels. The test results of repeatability of one taxel and sensitivity variation among 17 taxels are shown in Fig. 7(a) and 7(b). The result in Fig. 7(a) demonstrates that each taxel shows good performance in terms of repeatability 
(a dispersion of 5 increasing data), hysteresis (a difference between increasing and decreasing data), and zero return error (a difference between increasing and decreasing data at zero load), which is measured at a value smaller than $3 \%, 3 \%$ and $1 \%$ of the rated output, respectively.

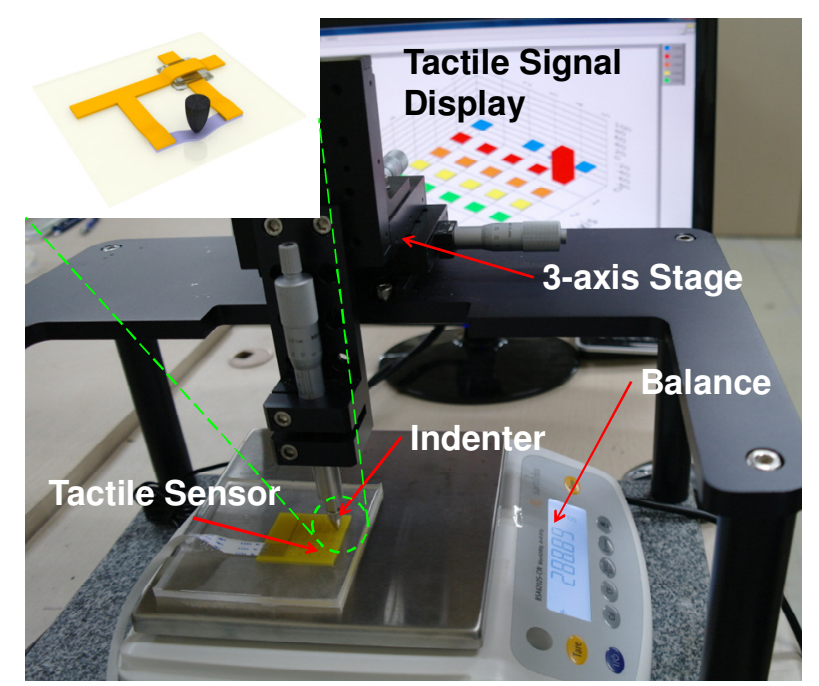

Fig. 6. Experimental setup for testing the force-distribution sensing device.
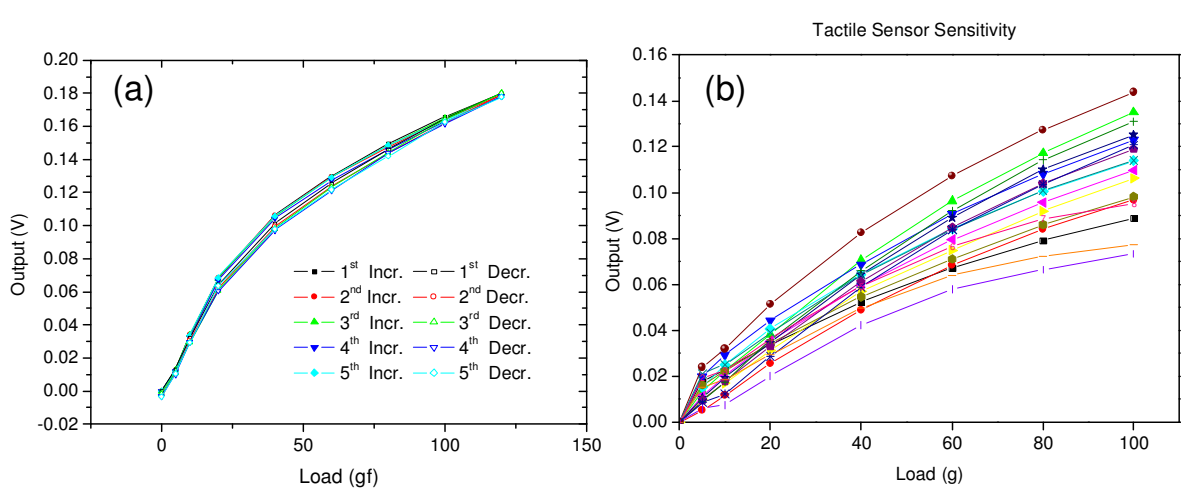

Fig. 7. Test results of the force-distribution sensing device: (a) repeatability, hysteresis test of single taxel, (b) test results of 17 taxels showing dispersion of sensitivity.

Fig. 7(b) shows the responses of 17 taxels. Each graph represents an average of five loading curves for each taxel. We observed a sensitivity variation of less than $\pm 30 \%$ (one standard deviation) among the 17 taxels. Such variation is thought to originate partially from the inherent dispersion of the gage factors among the 17 semiconductor strain gages. We suspect that a variation of the tip position relative to gauges might be attributable to such variation as well. Each gauge has $0.5 \mathrm{~mm}$ in length so that it is difficult to position the tip accurately at the centre of each gauge with manual control. 
Adding a load accepting structure like a bump on each gauge could reduce such a variation due to incomplete positioning.

\subsection{Test of the temperature sensor}

The temperature sensor was tested by soaking it into a bath filled by water. In the bath, a reference temperature sensor with a high resolution of $0.1^{\circ} \mathrm{C}$ was installed for measuring temperature of water. The temperature was changed from approximately $0{ }^{\circ} \mathrm{C}$ to $30{ }^{\circ} \mathrm{C}$ with $1{ }^{\circ} \mathrm{C}$ step. This procedure was repeated five times to determine the repeatability. The result of calibration is shown in Fig. 8. The relationship between relative resistance change and temperature is quite linear. The slope of the fitted line is determined to be $0.32 \% / \mathrm{K}$, which is close to the value of temperature coefficient of resistance of bulk copper $0.39 \% / \mathrm{K}$. The copper used in the temperature sensor is a deposited metal, not a bulk metal so that such a slight difference is reasonable. The resistance change was quite repeatable during five measurements. The standard deviation was measured to be less than $0.3^{\circ} \mathrm{C}$.

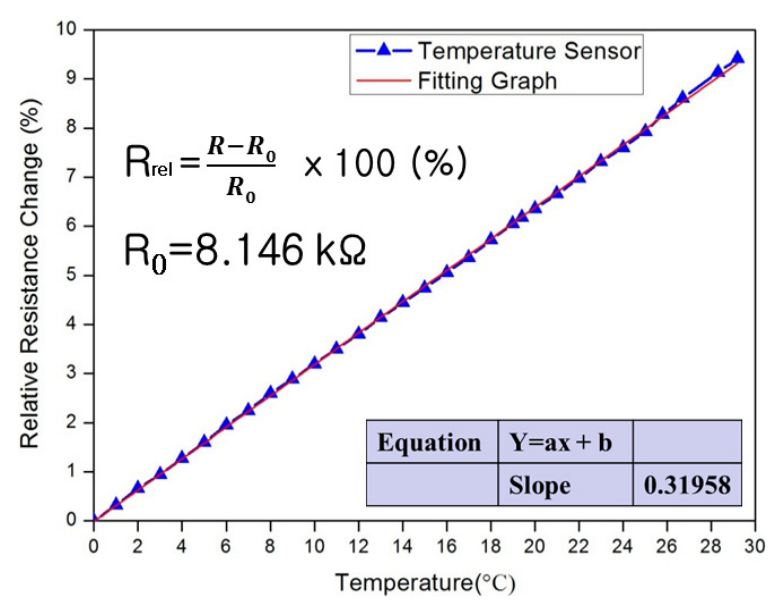

Fig. 8. Calibration result of the flexible temperature sensor.

\section{Conclusion}

We fabricated a flexible tactile sensor measuring force-distribution and temperature simultaneously in order to demonstrate that our new approach is potentially capable of simultaneously achieving three major goals for tactile sensors, that is, high performance, flexibility, and robustness. The concept of our approach is to use totally different two materials together-inorganic silicon and polymers-of which the properties are complementary to each other. Using thin and highly-doped single-crystal silicon ribbons as sensing elements can provide high-sensitivity and repeatability while using polymers with no diaphragm or no membrane as spring elements can give more mechanical robustness along with flexibility. 
Such an approach was successfully demonstrated through a series of test with a prototype tactile sensor consisting of micro-structured semiconductor strain gages, deposited metals ( $\mathrm{Au}$ for signal lines and $\mathrm{Cu}$ for the temperature sensor) and polymers (PDMS and polyimide). The prototype tactile sensor showed good performance on force measurements, with repeatability better than $3 \%$, hysteresis less than $3 \%$, and zero-return error less than $1 \%$ and also on temperature measuring characteristics showing good linearity and accuracy of $0.3{ }^{\circ} \mathrm{C}$, all of which suggest that our approach can be used for high performance flexible tactile sensors.

Based on our approach, we will proceed to develop more sophisticated tactile sensors that have measuring capabilities of 3 -axis force and vibration up to $250 \mathrm{~Hz}$ with on-board signal processing circuits. We hope they can be used to measure and recognize the texture of various objects in robotic systems.

\section{Acknowledgments}

This research was supported in part by the Converging Research Center Program through the Ministry of Science, ICT and Future Planning, Korea (2013K000331) and by the Industrial Strategic Technology Development Program (10041066, development of tactile information storing and playing platform based on flexible sensors for immersive media) funded by the Science, ICT and Future Planning, Korea.

\section{References}

1. R. S. Dahiya, G. Metta, M. Valle, and G. Sandini, IEEE T. Robot. 26, 1 (2010).

2. V. Maheshwari and R. Saraf, Angew. Chem. Int. Ed. 47, 7808 (2008).

3. H. Yousef, M. Boukallel, and K. Althoefer, Sensor Actuat. A-Phys. 167, 171 (2011).

4. M.-S. Kim, S.-J. Shin, and Y.-K. Park, Proc.of APMF 2011(Xian, China), pp. 68 (2011).

5. M.-S. Kim, S.-J. Shin, and Y.-K. Park, Int. J. Prec. Eng. Manuf. 13, 1941 (2012).

6. M. A. Meitl, Z. T. Zhu, V. Kumar, K. J. Lee, X. Feng, Y. Y. Huang, I. Adesida, R. G. Nuzzo, and J. A. Rogers, Nat. Mater. 5, 33 (2006). 